PROCEEDINGS OF THE

AMERICAN MATHEMATICAL SOCIETY

Volume 46, Number 3, December 1974

\title{
THE HEIGHTS OF FORMAL $A$-MODULES
}

\author{
WILLIAM C. WATERHOUSE ${ }^{1}$
}

ABSTRACT. Let $A$ be a discrete valuation ring, finite over $\mathrm{Z}_{p}$, acting on a commutative formal Lie group of height $h$. Then $h$ is a multiple of $\left|A: Z_{p}\right|$; and if $A$ acts on the tangent space by scalar multiplications, the dimension of the group is at most $h /\left|A: Z_{p}\right|$.

Theorem 1. Let $k$ be a local ring of residue characteristic $p$. Let $G$ be a commutative formal Lie group over $k$ of finite height $h$. Let $A$ be the ring of integers in a finite extension $K$ of $\mathbf{Q}_{p}$, and suppose $A$ acts as endomorphisms of $G$. Then $h$ is divisible by $\left|K: \mathbf{Q}_{p}\right|$.

Proof. Both $h$ and the existence of $A$-action are unaffected by base change, so we may assume $k$ is an algebraically closed field. Let $W$ be the ring of Witt vectors over $k$; then $G$ corresponds to a Dieudonné module $M$, a module over $W[F, V]$ which is free of rank $b$ over $W$. Since $A$ acts on $G$, it acts on $M$, which is thus a module over the $\mathbf{Z}_{p}$-tensor product $A \otimes W$. The following lemma then completes the proof.

Lemma. $M$ is a free module over $A \otimes W$.

Proof. Let $L$ be the fraction field of $W$. Let $E$ be the maximal unramified subextension of $K$, and $B$ its ring of integers. Let $\sigma$ be the Frobenius automorphism. Then $B \otimes B$ is isomorphic to $B \times \cdots \times B$ via $b \otimes b^{\prime} \mapsto$ $\left\langle b \sigma^{r}\left(b^{\prime}\right)\right\rangle$, since $E$ is unramified; and id $\otimes \sigma$ is an automorphism of $B \otimes B$ whose powers interchange all the factors of the direct product.

Since $k$ is algebraically closed, there is a copy of $B$ in $W$, and $B \otimes W$ is similarly isomorphic to $W \times \cdots \times W$. It follows then that

$$
A \otimes W \simeq A \otimes_{B} B \otimes W \simeq\left(A \otimes_{B} W\right) \times \cdots \times\left(A \otimes_{B} W\right),
$$

and id $\otimes \sigma$ still interchanges the factors. Now $K$ is totally ramified over $E$, so $A$ is generated over $B$ by an Eisenstein element. Since $L$ is unramified over $E$, the same element is still Eisenstein over $W$; hence $K \otimes_{E} L$ is a field and $A \otimes_{B} W$ is the valuation ring in it.

Received by the editors September 21, 1973.

AMS (MOS) subject classifications (1970). Primary $14 \mathrm{~L} 05$.

1 Supported by NSF contract GP-25600.

Copyright $\odot$ 1974, American Mathematical Society 
Thus the $A \otimes W$-module $M$ splits into the direct sum of modules over the various valuation ring factors $A \otimes_{B} W$; these modules are free since they are finitely generated and torsion-free. To prove $M$ itself is free it is enough to show the summands all have the same rank. Let $e$ be the primitive idempotent for one of the factors, so that $e M$ is the corresponding summand. For any $m$ in $M$ we have $F(e m)=[(\mathrm{id} \otimes \sigma) e] F m$, so $F$ takes $e M$ to $[(\mathrm{id} \otimes \sigma) e] M$. Thus the powers of $F$ map all the summands of $M$ to each other. But $V F=p$, so $F$ is injective on $M$, and hence the summands must all have the same rank.

This theorem is no surprise; it generalizes a result which was known (with a different proof) in the one-dimensional case [4, p. 470]. The Lemma, though more technical, is of more interest; for it also implies the following result, which was conjectured by Lubin.

Theorem 2. Assume the hypotheses of Theorem 1. Assume also that $G$ is a formal A-module in the sense of [5], i.e. the action which each element of $A$ induces on the tangent space of $G$ is a scalar multiplication. Then $\operatorname{dim} G \leq h /\left|K: \mathbf{Q}_{p}\right|$.

Proof. Again base change allows us to assume $k$ is an algebraically closed field, so we have a Dieudonné module $M$ which we know satisfies the Lemma. We also know that $M / V M$ is the tangent space of $G$, and its $k$-dimension is $\operatorname{dim} G$. (This assumes we are working with covariant Dieudonné modules, as in [3] or [1]. A similar argument will go through for the contravariant modules of [2].) Let $\left\{m_{i}\right\}$ be a basis of $M$ as an $A \otimes W$-module. Then the images of the $m_{i}$ are generators of the module $M / V M$. The elements of $W$ act on this tangent space via scalar multiplications; by assumption the elements of $A$ do so also, and thus the entire $A \otimes W$-action is via scalar multiplications. Hence the generators over $A \otimes W$ are generators over $k$, and the number of them is at least as large as the dimension.

\section{REFERENCES}

1. P. Cartier, Relèvement des groupes formels commutatifs, Séminaire Bourbaki: 1968/69, Exposé 359, Lecture Notes in Math., vol. 179, Springer-Verlag, Berlin and New York, 1971. MR 42 \#7460.

2. M. Demazure, Lectures on p-divisible groups, Lecture Notes in Math., vol. 302, Springer-Verlag, New York, 1972.

3. J. Dieudonné, Lie groups and Lie.hyperalgebras over a field of characteristic p> 0. IV, Amer. J. Math. 77 (1955), 429-452. MR 17, 174. 
4. J. Lubin, One-parameter formal Lie groups over p-adic integer rings, Ann. of Math. (2) 80 (1964), 464-484. MR 29 \#5827.

5. - Formal A-modules defined over A, Symposia Mathematica, Vol. III (INDAM, Rome, 1968/69), Academic Press, London, 1970, pp. 241-245. MR 42 \#260.

DEPARTMENT OF MATHEMATICS, CORNELL UNIVERSITY, ITHACA, NEW YORK 14850 